\title{
Three Narcoleptics with Restless Leg Syndrome
}

\author{
Gothi Dipti ${ }^{1}$, Agarwal Mohit ${ }^{2}$, Deshmukh Ishani ${ }^{3}$
}

\begin{abstract}
We are reporting the third largest Indian narcolepsy case series. Among 250 consecutive patients referred to our sleep center for polysomnography (PSG), 3 of them were diagnosed to have narcolepsy with cataplexy (NC). They had history of excessive daytime sleepiness (EDS) and sudden loss of muscle power leading to fall without alteration in consciousness. Their polysomnographies did not have obstructive sleep apnea (OSA). All of them had multiple sleep latency test (MSLT) showing sleep latency of less than 8 minutes and two sleep-onset rapid eye movement period (SOREMP). Since narcolepsy patients commonly have restless leg syndrome (RLS), RLS was studied in detail in them. All our patients with NC had RLS. The patients from Western countries with NC and RLS have normal or high iron level. But the serum iron level was low in two patients and normal in one patient. The management of RLS secondary to iron deficiency is thus an important component of NC in Indian patients.

Key messages:

- Clinical history is very important for suspicion of narcolepsy.

- Multiple sleep latency test is diagnostic of narcolepsy in the current clinical context.

- Restless leg syndrome is diagnosed on history alone. Since it occurs commonly with narcolepsy, history of RLS in important in narcolepsy patients.

- Iron deficiency can also be causative factor for RLS in narcolepsy in Indian patients though it is reported to be due to narcolepsy itself in Western counterparts.

- Modafinil alone is not useful in the treatment of narcolepsy; a tailor-made pharmacological and supportive non-pharmacological approach is essential.

Keywords: Iron deficiency, Narcolepsy with cataplexy, Polysomnography, Restless leg syndrome.

Indian Journal of Sleep Medicine (2020): 10.5005/jp-journals-10069-0049
\end{abstract}

\section{INTRODUCTION}

Narcolepsy is characterized by excessive daytime sleepiness (EDS) and symptoms related to abnormal regulation of wakefulness and sleep with or without cataplexy. ${ }^{1}$ The occurrence of restless legs syndrome (RLS) in narcolepsy with cataplexy (NC) is common. ${ }^{2}$ However, there is no study assessing the comorbidity of RLS in NC from India. We are reporting the third largest case series of NC. We studied RLS in all three patients. The study is about description of all three patients and their management.

\section{Case Description}

We evaluated sleep clinic data of 250 patients referred to pulmonary, sleep, and critical care medicine department from March 2016 to November 2019 for polysomnography (PSG). Three patients were diagnosed to have NC. Their clinical history, demographics, and investigations are as follows and in Tables 1 and 2 .

Three patients aged 27, 39, and 51 years, two of them women and one man were referred for EDS and sudden loss of muscle power leading to fall without alteration in consciousness, headache, or sensory disturbances. Two of them had hypnagogic hallucination. One of them had insomnia and depression. All the three patients had abnormal sensation in leg at rest in the evening, improving with movement suggestive of RLS. Their average duration of symptoms was 6 years. One of the women was threatened divorce. The other woman had been to a neurologist but not diagnosed. The man had motor vehicular accidents twice due to sleepiness.

Their Epworth Sleepiness Scale (ESS), PSG, MSLT, and iron profile data are given in Table 3. All three patients were given non-pharmacological and pharmacological agents (modafinil) for NC. But the first one improved only after addition of oral iron
${ }^{1-3}$ Department of Pulmonary Medicine, ESI-Postgraduate Institute of Medical Sciences and Research, Basai Darapur, New Delhi, India

Corresponding Author: Gothi Dipti, Department of Pulmonary Medicine, ESI-Postgraduate Institute of Medical Sciences and Research, Basai Darapur, New Delhi, India, Phone: +91 9971550550, e-mail: diptigothi@gmail.com

How to cite this article: Dipti G, Mohit A, Ishani D. Three Narcoleptics with Restless Leg Syndrome. Indian J Sleep Med 2020;15(1):5-8.

Source of support: Nil

Conflict of interest: None

supplement and pramipexole $0.25 \mathrm{mg}$. The second patient required additional fluoxetine (selective serotonin reuptake inhibitor) for depression. The third patient required additional methylphenidate and iron therapy for symptom relief. None of our patient required anticataplectic drugs as they had mild cataplexy which was improved on education, good sleep hygiene practice, and control over strong emotions. ${ }^{3}$

\section{Discussion}

Narcolepsy with cataplexy is seen in 0.025 to $0.05 \%$, while narcolepsy without cataplexy is observed in 0.02 to $0.034 \%$ of adults worldwide. In Japanese population, the prevalence of narcolepsy is $0.16 \%$ highest, whereas Jewish and Saudi Arabian populations have the prevalence of $0.010 \% .{ }^{4}$ There is no prevalence data from India. The world wide prevalence of RLS among the general population is 5 to $15 \%$ and that from India is 2.9 to $15 \% .^{5}$ Restless leg syndrome is significantly more prevalent among NC patients (14.7\%) than in 
Table 1: Demographic, anthropometric, and clinical characteristics of the patients

\begin{tabular}{llll}
\hline & Patient 1 & Patient 2 & Patient 3 \\
\hline Age (years)/sex & $29 / \mathrm{F}$ & $39 / \mathrm{F}$ & $51 / \mathrm{M}$ \\
EDS & + & + & + \\
Addiction (smoking/alcohol/substance abuse) & No & No & No \\
Occupation & Homemaker & Homemaker & Driver \\
Cataplexy & + & - & + \\
Hypnagogic hallucinations & - & + & + \\
Insomnia & - & + & - \\
Depression & - & + & - \\
RLS & + & + & + \\
Family history & Not significant & Not significant & Not significant \\
General examination & & & \\
$\quad$ Weight $(k g)$ & 35 & 65 & 56 \\
Height (m) & 1.45 & 1.53 & 1.70 \\
BMI (kg/m ${ }^{2}$ ) & 16.6 & 27.7 & 19.8 \\
Temperature & Afebrile & Afebrile & Afebrile \\
Pulse rate & $78 /$ minutes & $76 /$ minutes & $96 /$ minutes \\
Blood pressure (mm Hg) & $104 / 54$ & $112 / 68$ & $100 / 70$ \\
Respiratory rate & $18 /$ minutes & $16 /$ minutes & $18 /$ minutes \\
Oxygen saturation at room air (\%) & 96 & 98 & 95 \\
Systemic examination & Normal & Normal & Normal \\
\hline
\end{tabular}

F, female; M, male; BMI, body mass index; EDS, excessive daytime sleepiness; RLS, restless legs syndrome

Table 2: Blood investigations

\begin{tabular}{lccc}
\hline & Patient 1 & Patient 2 & Patient 3 \\
\hline Biochemical investigations & & & \\
Blood urea nitrogen $(\mathrm{mg} / \mathrm{dL})$ & 24 & 17 & 28 \\
Serum creatinine $(\mathrm{mg} / \mathrm{dL})$ & 0.6 & 0.9 & 1.2 \\
Random blood sugar $(\mathrm{mg} / \mathrm{dL})$ & 92 & 86 & 79 \\
Serum cholesterol $(\mathrm{mg} / \mathrm{dL})$ & 165 & NA & 156 \\
Serum triglycerides $(\mathrm{mg} / \mathrm{dL})$ & 83 & NA & 117 \\
Serum HDL $(\mathrm{mg} / \mathrm{dL})$ & 56 & NA & 54 \\
Serum TSH $(\mathrm{mlU} / \mathrm{L})$ & 1.22 & 1.5 & 1.2 \\
Hematological investigations & & & \\
Hemoglobin $(\mathrm{g} / \mathrm{dL})$ & 12.3 & 11.9 & 10.6 \\
Total leukocyte count $(\mathrm{cells} / \mu \mathrm{L})$ & 4500 & 9600 & 5700 \\
Platelets $(\mathrm{lakhs} / \mu \mathrm{L})$ & 1.8 & 1.11 & 4.51 \\
Serum iron $(\mu \mathrm{g} / \mathrm{dL})$ & 118 & 92 & 40 \\
Serum ferritin $(<50 \mathrm{ng} / \mathrm{mL})$ & 26 & 86 & 13 \\
Serum TIBC $(\mu \mathrm{g} / \mathrm{dL})$ & 477 & 204 & 277 \\
\hline
\end{tabular}

$\mathrm{HDL}$, high-density lipoprotein; TSH, thyroid-stimulating hormone; TIBC, total iron-binding capacity; NA, not applicable

controls (3.0\%). ${ }^{2}$ So, there is a causative link between NC and RLS. We found RLS in all our patients of NC.

Narcolepsy is a clinical syndrome of daytime sleepiness, cataplexy, hypnagogic hallucinations, and sleep paralysis. Cataplexy is a sudden loss of muscle control, typically on both sides of the body, triggered by strong emotions. ${ }^{6}$ The cardinal symptom of daytime sleepiness occurs in every patient, whereas other symptoms such as cataplexy, hypnagogic hallucinations, and sleep paralysis are reported in 70,25 , and $5 \%$ of patients, respectively. ${ }^{7}$ It is caused by a loss of neurons that produce orexin neuropeptides. Though the exact cause is not known, it has human leukocyte antigen (HLA) link with the class II DQB1*06:02 allele. The described reports suggest that precipitating factors like respiratory tract infection, injury to orexin-producing neurons secondary to sarcoidosis, demyelination or stroke, tumor, and paraneoplastic syndrome lead to NC. ${ }^{6}$

Multiple sleep latency test (MSLT) is essential for the diagnosis of NC. Multiple sleep latency test consists of five nap opportunities, each of 20 minutes duration, performed at 2-hour intervals. Multiple sleep latency test assessment should include latency from lights out to the first epoch of sleep. The absence of sleep in the nap opportunity is recorded as sleep latency of 20 minutes. The mean sleep latency and the number of sleep onset REM periods (SOREMPs) are useful in diagnosing narcolepsy. ${ }^{8}$

The diagnostic criteria for narcolepsy have been described in sleep disorder guidelines (International Classification of Sleep Disorders - third edition). As per the guidelines, MSLT showing a mean sleep latency of $\leq 8$ minutes and $\geq 2$ minutes SOREMPs are diagnostic of narcolepsy provided other causes of EDS are ruled out. Narcolepsy is divided into type I and type II. If the patient has cataplexy or cerebrospinal fluid hypocretin-1 concentration $>110$ $\mathrm{pg} / \mathrm{mL}$ or $>1 / 3$ of mean values obtained in normal subjects it is called type I narcolepsy or NC. Our patients had type I narcolepsy because of the presence of cataplexy. Cerebrospinal fluid hypocretin was not required because of cataplexy. ${ }^{9}$

Restless leg syndrome is diagnosed with four essential criteria that can be abbreviated as "URGE," i.e., urge to move, rest induced, gets better with activity, and evening and night accentuation. In total, $90 \%$ of RLS patients have periodic limb movements during sleep (PLMS). Restless leg syndrome requires a history for diagnosis, whereas PLMS requires PSG for diagnosis. ${ }^{10}$ Various pathophysiological mechanisms like genetic variants, abnormal iron metabolism, dopaminergic dysfunction, and central opiate system involvement have been implicated in the occurrence of RLS/ 
Three Narcoleptics with Restless Leg Syndrome

Table 3: Polysomnography and MSLT data of patients with narcolepsy

\begin{tabular}{llll}
\hline & Patient 1 & Patient 2 & Patient 3 \\
\hline ESS & $17 / 24$ & $24 / 24$ & $17 / 24$ \\
STOP-Bang score & $1 / 8$ & $1 / 8$ & $3 / 8$ \\
Polysomnographic findings & & & \\
$\quad$ Total sleep time & 212 & 373.5 & 135 minutes \\
Sleep efficiency & 62.9 & 88.5 & $43.3 \%$ \\
Sleep latency & 30 & 6 & 58.5 minutes \\
AHI (events/hour) & 0.6 & 0.8 & 4.4 \\
Desaturation index (events/hour) & $1.1 /$ hour & $5.8 /$ hour & $4.9 /$ hour \\
Baseline SpO 2 & $98 \%$ & $95 \%$ & $95 \%$ \\
Minimum SpO & & $92 \%$ & $91 \%$ \\
Total limb movements & $94 \%$ & 797 & 490 \\
Total PLMS & 622 & 516 & 456 \\
Total PLMS arousals & 499 & 11 & 15 \\
Limb movement index & 23 & 128 & 217.8 \\
PLMS index & 176 & 82.9 & 202.7 \\
PLMS arousal index & 141.2 & 1.8 & 6.7 \\
MSLT findings & 6.5 & & 1 minute 15 seconds \\
Sleep latency (mean) & 1 minute 16 seconds & 2 minute 57 seconds & 4 \\
SOREMs & 2 & 3 & \\
\hline
\end{tabular}

ESS, Epworth sleepiness scale; STOP-Bang, snoring, tiredness, observed apnea, high BP, BMI, age, neck circumference, and male gender; $\mathrm{AHI}$, apnea hypopnea index; $\mathrm{SpO}_{2}$, oxygen saturation targeting by pulse oximetry; MSLT, multiple sleep latency test; SOREMs, sleep onset-rapid eye movements; PLMS, periodic limb movements during sleep

PLMS. Iron is a cofactor for tyrosine hydroxylase, the rate-limiting enzyme involved in the synthesis of dopamine. An abnormal iron metabolism leads to a decreased production of dopamine, thereby leading to symptoms of RLS/PLMS. ${ }^{11}$ The nature of association between NC and RLS/PLMS is thought to be due to common pathophysiology involving dopaminergic pathways directly as the patients with RLS and NC having normal or high serum iron levels. ${ }^{5}$ All the patients in our case series suffered from RLS and PLMS, but one had normal iron levels, whereas other two had it secondary to low iron levels. Thus, in patients with NC, it is important to rule out RLS secondary to iron deficiency rather than assume that it is secondary to NC.

The diagnosis of $\mathrm{NC}$ is usually made only after serious problems have arisen such as declining grades at school, poor performance at work, body injury, or motor vehicle accident. Our patients were pushed to the verge of divorce, disturbed family, and road traffic accident, respectively, before getting medical attention. Only 28 cases have been reported from India. The largest series has been of 20 patients, ${ }^{12}$ and the second largest series has been of 4 patients. ${ }^{3}$ Ours is the third largest series. Restless leg syndrome/PLMS, a common co-occurrence has not been evaluated earlier from any of the series/case reports from India. All three patients in this case series had symptoms of narcolepsy for more than 5 years, but they were misdiagnosed and mismanaged. So, it is important to increase the awareness of the disease.

The pharmacological agent modafinil is the first-line therapy in the treatment of EDS in narcolepsy, but modafinil alone is not useful. Non-pharmacological therapies such as napping, good sleep hygiene, and avoidance of drugs that can worsen daytime sleepiness are equally important. The second-line therapy for daytime sleepiness is methylphenidate. ${ }^{13}$ One of our patients required methylphenidate for control of EDS. Sodium oxybate and venlafaxine are indicated if cataplexy symptoms are uncontrollable with supportive management. ${ }^{12}$ Sodium oxybate is not available in India. Fortunately, none of our patients required sodium oxybate. Antidepressant like fluoxetine may be needed in patients with depression, like one of our patients. Restless leg syndrome if due to NC may not require additional treatment, but if it is due to iron deficiency, iron supplements are essential.

To conclude, whenever a patient presents to physician with excessive sleepiness, without history suggestive of OSA, narcolepsy must be suspected. Multiple sleep latency test is an important investigative tool for the diagnosis of NC. Restless leg syndrome must be looked for and addressed appropriately. Modafinil alone is not adequate for the treatment of NC.

\section{REFERENCES}

1. Sateia MJ. International classification of sleep disorders third edition Chest 2014;146(5):1387-1394. DOI: 10.1378/chest.14-0970.

2. Plazzi G, Ferri R, Dauvilliers Y. Narcolepsy and restless legs syndrome. Sleep 2010;33(5):689-694. DOI: 10.1093/sleep/33.5.689.

3. Barateau L, Dauvilliers Y. Recent advances in treatment for narcolepsy. Ther Adv Neurol Disord 2019;12:1-12. DOI: 10.1177/1756286419875622.

4. Ray A. A four year experience in narcolepsy from a sleep clinic at a tertiary care centre with a short review of contemporary Indian literature. Indian J Med Res 2018;148(6):748-751. DOI: 10.4103/ijmr. IJMR_888_16.

5. Yeh P,Walters AS, Tsuang JW. Restless legs syndrome: a comprehensive overview on its epidemiology, risk factors, and treatment. Sleep Breath 2012;16(4):987-1007. DOI: 10.1007/s11325-0110606-x.

6. Scammell TE. Narcolepsy. N Engl J Med 2015;373(27):2654-2662. DOI: 10.1056/NEJMra1500587. 
7. Gupta AK, Sahoo S, Grover S. Narcolepsy in adolescence-a missed diagnosis: a case report. Innov Clin Neurosci 2017;14(7-8):20-23.

8. Littner MR, Kushida C, Wise M, et al. Standards of practice committee of the american academy of sleep medicine. Practice parameters for clinical use of the multiple sleep latency test and the maintenance of wakefulness test. Sleep 2005;28(1):113-121. DOI: 10.1093/sleep/28.1.113.

9. Reading PJ. Update on narcolepsy. J Neurol 2019;266(7):1809-1815. DOI: 10.1007/s00415-019-09310-3.

10. Gothi D. Sleep disorders in chronic obstructive pulmonary disease. Indian J Sleep Med 2015;10(1):11-21.
11. Gonzalez LP, Malkani RG. Update on restless legs syndrome:from mechanisms to treatment. Curr Neurol Neurosci Rep 2019;19(8):54 DOI: 10.1007/s11910-019-0965-4.

12. Gupta A, Shukla G, Goyal V, et al. Clinical and polysomnographic characteristics in 20 North Indian patients with narcolepsy. A seven year experience from a neurology service sleep clinic. Neurol India 2012;60(1):75-78. DOI: 10.4103/0028-3886.93602.

13. Swick TJ. Treatment paradigms for cataplexy in narcolepsy: past, present, and future. Nature and Science of Sleep 2015;7:159-169. DOI: $10.2147 /$ NSS.S92140. 